\title{
HCV clearance patterns in saliva and serum of patients with chronic HCV infection under interferon plus ribavirin therapy
}

\author{
P. Diz Dios ${ }^{1}$, A. Castro $^{2}$, I. Rodríguez ${ }^{3}$, N. G. Reforma ${ }^{1}$, M. Castro ${ }^{1}$, M. Eirea $^{1}$, M. \\ Hermida $^{3}$
}

${ }^{1}$ School of Medicine and Dentistry, Santiago de Compostela University, Vigo;

${ }^{2}$ Department of Internal Medicine, Juan Canalejo Hospital, La Coruña;

${ }^{3}$ Institute of Health Sciences, La Coruña, Spain

\begin{abstract}
Statements of the problem: Hepatitis C virus (HCV)-RNA is often present in saliva of HCV-infected patients, with plasma viral load being the only known predictable factor. Interferon plus ribavirin therapy yields a sustained reduction in HCV viremia. This study aimed to assess the presence of HCV in saliva and serum specimens from patients undergoing this combination therapy (CT).

Method of study: Paired serum and saliva specimens were collected from 44 chronic HCV-infected patients at basal time, 4 and 12 weeks after CT onset, at the end of treatment and 6 months latter. Serum HCV-RNA levels were determined by the polymerase chain reaction (PCR) Amplicor system. Presence of HCV-RNA in saliva was tested by a highly sensitive non-commercialized nested-PCR.

Results: The HCV-RNA was detected in 26 saliva specimens at basal time $(59.1 \%)$. In $34.1 \%$ of cases, a concordance viral clearance pattern in serum and saliva was observed in both responders (pattern 1a) and nonresponders (pattern $1 \mathrm{~b})$. In pattern $2(13.6 \%$ of cases), HCV was detected longer during CT in serum than in saliva (pattern 2a) or in saliva than in serum (pattern 2b). In $11.3 \%$ of patients, viral clearance was corroborated either in their serum (pattern 3a) or in their saliva (pattern 3b), but not in both fluids. Of the eight primary responders with 1a clearance pattern, seven were sustained responders. None of the patients with $2 \mathrm{a}$ clearance pattern was a sustained responder. Of the two primary responders showing the $3 \mathrm{~b}$ salivary pattern, one had already relapsed in the first 6 months of follow up.

Conclusions: The present results suggest that the monitoring of salivary levels of HCV would be a helpful means of determining sustained antiviral effects of interferon and ribavirin in the treatment of HCV disease.
\end{abstract}

Keywords: Hepatitis C virus; Interferon; Saliva 


\section{Introduction}

Saliva of patients with chronic hepatitis $C$ has been examined in several series for the presence of the hepatitis $\mathrm{C}$ virus (HCV) genome (HCV-RNA) by reverse transcription polymerase chain reaction (RTPCR), with percentages which vary between 0 (1) and $100 \%$ (2). We have studied the presence of HCVRNA in saliva from serum HCV-RNA-positive patients by a highly sensitive PCR method. RNA viral particles were detected in saliva specimens in more than $50 \%$ of $\mathrm{HCV}$-infected patients, with serum viral load being the only known predictable factor (3). The fact that HCV-RNA can be present in the saliva of patients with chronic hepatitis $\mathrm{C}$ provides a biologic basis for the potential non-sexual and nonpercutaneous transmission of $\mathrm{HCV}$ infection. Although some authors have suggested the importance of $\mathrm{HCV}$ contaminated saliva in the intrafamilial propagation of the infection (4) it has not been possible to determine their infective capacity.

Well-defined and standardized criteria for response to antiviral therapy have been established (5). These include circulating HCV-RNA, biochemical, and histologic responses both at the end of treatment and 6 months latter. However, antiviral effects of therapy in sites other than liver and blood have not been documented. In 1995, Roy et al. (6) studied saliva samples from HCV-positive patients under interferon (IFN) $\alpha-2 a$, suggesting that the role of salivary investigations in monitoring antiviral treatment was promising. Nowadays, IFN $\alpha$-2b plus ribavirin regimen represent a first-line treatment for patients with chronic hepatitis C (7). The aim of this study was to investigate the HCV clearance patterns in saliva and serum of patients with chronic hepatitis $\mathrm{C}$ under IFN plus ribavirin therapy.

\section{Material and methods}

The study population included 60 patients with chronic HCV infection, recruited amongst those referred for treatment to the Internal Medicine Department of Juan Canalejo Hospital (La Coruña, Spain). The selection of patients was carried out according to the following inclusion criteria: 18-60 years of age; presence of HCV-RNA in serum confirmed by nested-RT-PCR; absence of other concomitant liver diseases; negative HIV testing; subjects stated that they were neither habitual alcohol consumers nor recreational drug users.

All patients were treated with IFN $\alpha-2 b$ ( 3 MU thrice weekly) and ribavirin ( $800 \mathrm{mg}$ daily). Treatment lasted either 24 or 48 weeks, following the guidelines proposed at the 'International Consensus Conference on Hepatitis C' held in Paris in 1999 (5). In patients who relapsed or did not respond previously to IFN alone treatment was also extended to 48 weeks (8).

Paired serum and saliva samples were collected from every patient at baseline, 4 and 12 weeks after treatment onset, at the end of treatment and 6 months latter. Serum HCV-RNA levels were measured using a commercially available quantitative RT-PCR assay (Amplicor HCV Monitor, Roche Molecular Systems, Barcelona, Spain). HCV genotypes in serum samples were tested by a line probe assay (INNOLIPA HCV, Innogenetics NV, Zwijnaarde, Belgium). Stimulated whole saliva was collected applying the spitting method (9) and samples were stored in a sterile container at $-80^{\circ} \mathrm{C}$. Saliva specimens were evaluated for HCV-RNA amplifying a fragment of $251 \mathrm{bp}$ from the 5'-UTR region of the HCV, using the 209 and 939 primers (Life Technologies, Barcelona, Spain) as it has been previously described (3). Samples with macroscopically detectable blood were not included in the study. A Hem-Check-1 kit based in monoclonal antibodies (Menarini Diagnostics, Barcelona, Spain) was used in order to search for the presence of hemoglobin (hidden blood).

Primary responders were defined as patients who had normal aminotransferases and absence of circulating HCV-RNA at the end of treatment. When these conditions persisted more than 6 months after the cessation of therapy, the patients were considered sustained responders (5).

Ethical approval for the study was obtained from the Juan Canalejo Hospital Ethics' Committee. Written, informed consent was obtained from all patients. 


\section{Results}

Forty-four patients could complete treatment and undergo a 6-month follow up period. Twenty-one of them $(47.7 \%)$ where considered non-responders and $23(52.2 \%)$ fulfilled serologic criteria of primary responders. The other 16 patients did not finish treatment because of side effects (eight cases) or the follow up could not be completed (eight cases).

The HCV genotypes 1, 2, 3, and 4 were detected in the serum specimens. Genotype 1 was the most common $(1 \mathrm{a}=38.6 \% ; 1 \mathrm{~b}=11.3 \%)$, followed by genotypes $3 \mathrm{a}(15.9 \%), 4 \mathrm{c}$, and $4 \mathrm{~d}(11.3 \%)$.

The HCV was detected in saliva in 26 patients $(59.1 \%)$ before starting therapy. The other 18 patients $(40.9 \%)$ became classified as pattern 0 . Pattern 1 can be defined as a concordance viral clearance pattern between serum and saliva (Fig. 1). This pattern was observed in $34.1 \%$ of cases. After finishing treatment, the virus was undetectable in eight patients in serum and saliva (pattern 1a) while in seven cases it could be detected in both fluids (pattern 1b). Pattern 2a was defined as a late viral clearing in serum with regard to saliva (five cases). In pattern $2 \mathrm{~b}, \mathrm{HCV}$ was detected longer in saliva than in serum (one case). On the whole, pattern 2 was observed in $13.6 \%$ of the cases (Fig. 2). After finishing treatment, in five patients viral clearance could only be corroborated either in their serum (pattern 3a) or in their saliva samples (pattern 3b), but not in both fluids (Fig. 3). This different clearance viral pattern between serum and saliva (pattern 3) was observed in $11.3 \%$ of patients. Pattern 3a in which HCV remains present in saliva but not in blood in spite of antiviral therapy is the most relevant.

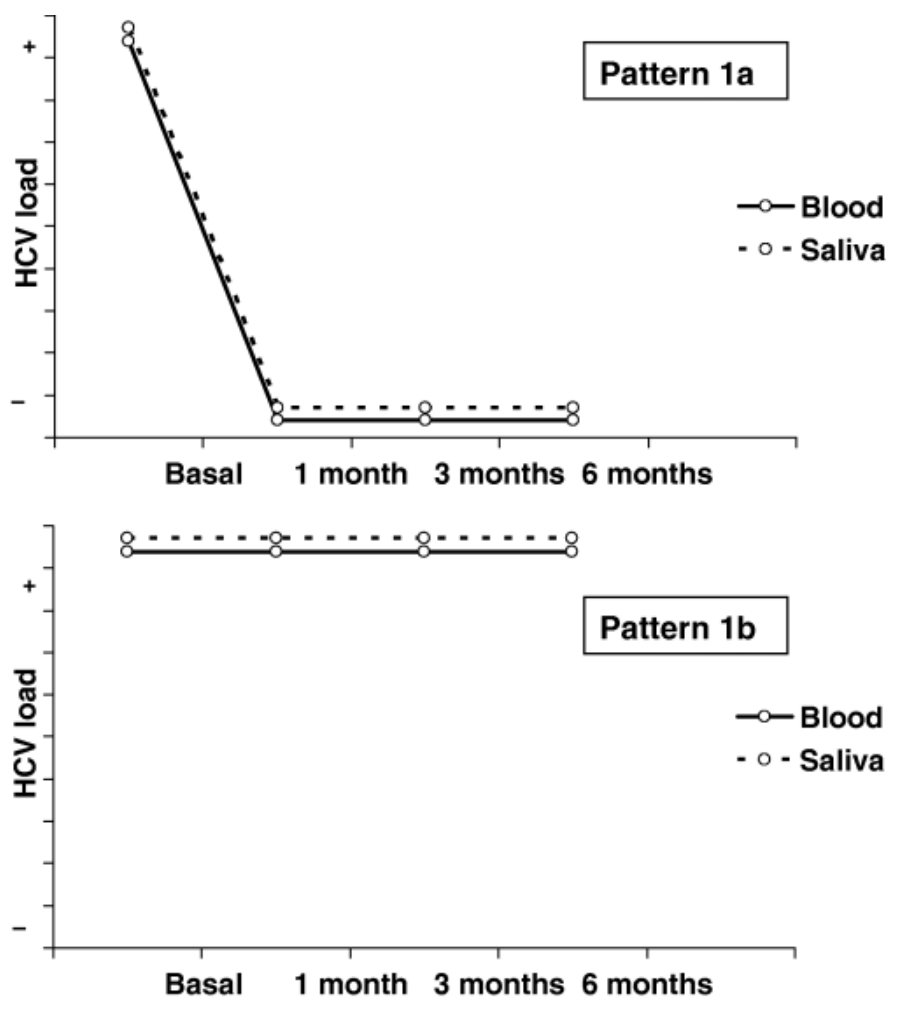

Figure 1. Pattern 1 or concordance viral clearance pattern (34.1\% of cases). After finishing treatment, the virus was undetectable in serum and saliva (pattern 1a) or it could be detected in both fluids (pattern 1b). 

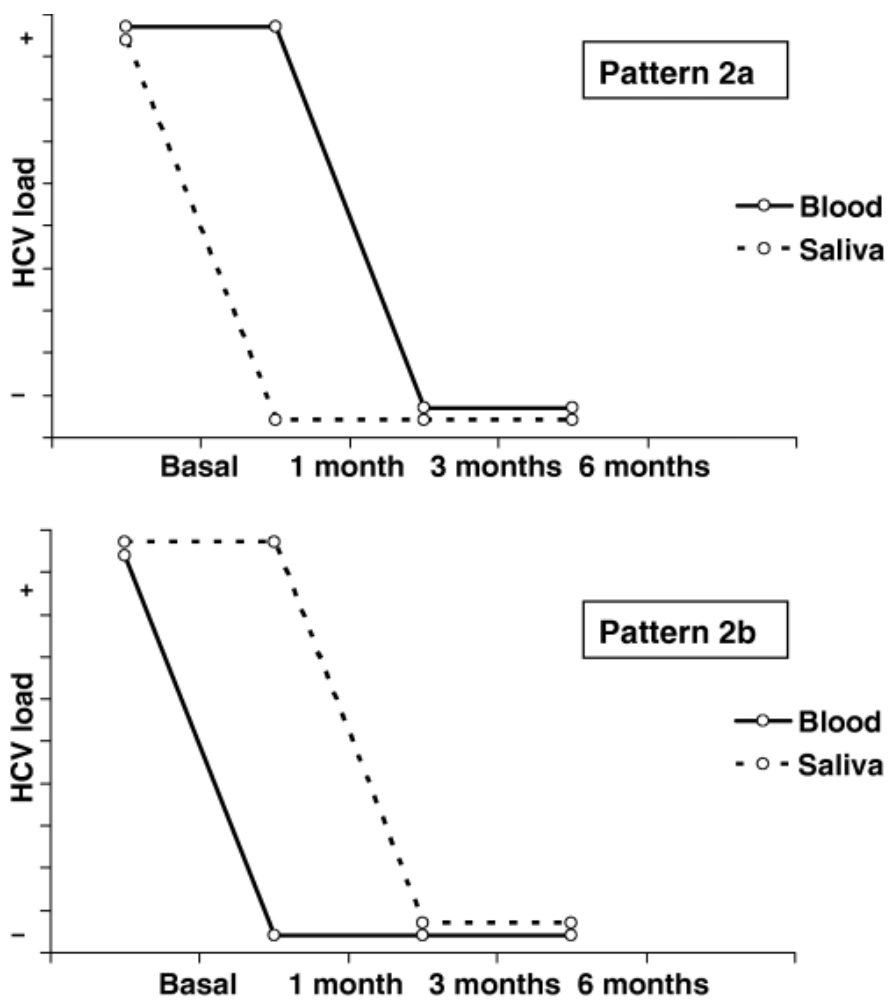

Figure 2. Pattern 2 or delayed viral clearance pattern (13.6\% of the cases). In pattern $2 \mathrm{a}$, there is a late viral clearing in serum with regard to saliva. In pattern $2 \mathrm{~b}$, hepatitis $\mathrm{C}$ virus (HCV) was detected longer in saliva than in serum.

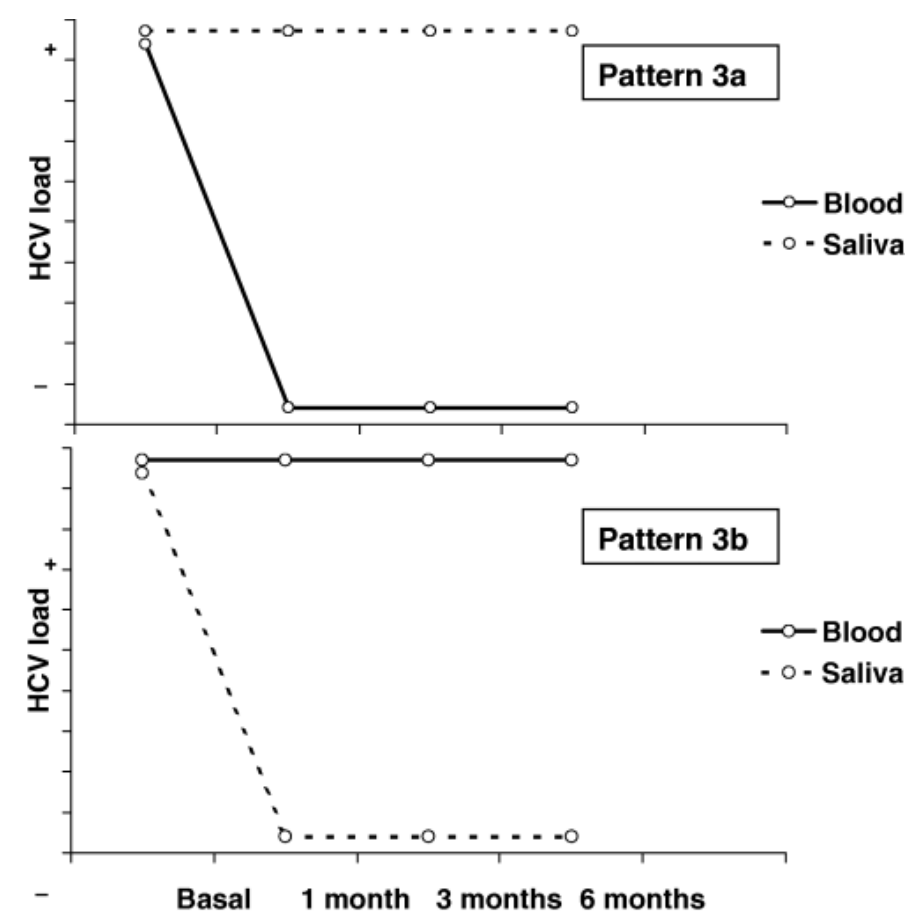

Figure 3. Pattern 3 or discordant pattern (11.3\% of cases). Viral clearance was corroborated either in serum (pattern $3 \mathrm{a}$ ) or in saliva (pattern $3 \mathrm{~b}$ ), but not in both fluids. 
The distribution of HCV genotypes in serum samples was not correlated with any viral clearance pattern in saliva following IFN plus ribavirin therapy.

After 6 months of follow up, 15 patients (34.5\%) were considered as sustained responders. The results of the serum response to treatment in relation to the HCV clearance pattern in saliva are detailed in Table 1. Of the eight primary responders with 1a salivary pattern, seven were sustained responders. None of the patients with $2 \mathrm{a} \mathrm{HCV}$ clearance pattern in saliva, was a sustained responder. Of the two primary responders showing the $3 \mathrm{~b}$ salivary pattern, one had already relapsed in the first 6 months of follow up.

Table 1. Serum response to interferon plus ribavirin therapy in relation to the HCV clearance pattern in saliva $(n=44)$

\begin{tabular}{llllr}
\hline & Non-responders $^{\mathrm{a}}$ & Primary responders $^{\mathrm{b}}$ & Sustained responders $^{\mathrm{c}}$ & Total \\
\hline Pattern 0 & 9 & 9 & 6 & 18 \\
Pattern 1a & 0 & 8 & 7 & 8 \\
Pattern 1b & 7 & 0 & 0 & 7 \\
Pattern 2a & 2 & 3 & 0 & 5 \\
Pattern 2b & 0 & 1 & 1 & 1 \\
Pattern 3a & 3 & 0 & 0 & 3 \\
Pattern 3b & 0 & 2 & 1 & 2 \\
Total & $21(47.7 \%)$ & $23(52.2 \%)$ & $15(34.5 \%)$ & \\
\hline
\end{tabular}

\footnotetext{
${ }^{\text {a }}$ Patients with abnormal aminotransferases or detectable hepatitis $\mathrm{C}$ virus (HCV)-RNA in serum at the end of treatment.

${ }^{\mathrm{b}}$ Patients with normal aminotransferases and absence of circulating HCV-RNA at the end of treatment.

${ }^{\mathrm{c}}$ Patients with normal aminotransferases and absence of circulating HCV-RNA persisting more than 6 months after the end of treatment
}

\section{Discussion}

In more than one half of the saliva samples collected before the beginning of treatment HCV-RNA was detected, this rate coinciding with other series in which the same probe assay was applied (3). In some series, virus particles have been only detected in the cellular fraction $(10,11)$. However, other authors, in agreement with our results, have isolated HCV-RNA from the pellet, from the supernatant or from both $(3,12)$, suggesting that HCV-RNA is not derived exclusively from cellular component.

It has been suggested that monitoring the effect of IFN by evaluating HCV-RNA in saliva has not shown a direct relationship with HCV in blood (6). In agreement with these authors, the description of three different HCV clearance patterns in saliva of patients with chronic HCV infection under interferon plus ribavirin therapy has been made.

In a previous study, the only variable we observed which conditioned the presence of HCV-RNA in saliva, was the serum viral load (3), justifying pattern 1 as prevailing. Pattern 2 a could also be interpreted as early clearance in saliva by diminishing the serum viral load under a certain level.

In order to explain patterns $2 b, 3 a$, and $3 b$, three hypotheses are now posed: the first one was the different sensitivity of HCV detection tests applied in saliva (10 copies/ml) (3) and serum specimens (600 IU/ml) (13). In a series published by Roy et al. (14), some HCV-RNA-seronegative patients were identified with detectable HCV-RNA in their saliva. The authors suggested that these individuals were viremic below the RT-PCR detection level in serum. Nevertheless, in the present study, HCV-RNAnegative serum tested by the Amplicor ${ }^{\circledR}$ assay were also negative with the nested-PCR used in the analysis of saliva samples. 
Another possibility was whether the pharmacodynamic of interferon and ribavirin could cause differences in the penetration and activity of this combination therapy (CT) in serum and saliva. In a paper by Roy et al. (6) HCV-RNA clearance was detected in the saliva of two treated patients who did not respond to IFN, which corresponds with our pattern $3 b$. Nevertheless, patterns $3 a$ and $3 b$ are contradictory, and could not be justified with this argument.

Finally, it could be argued that there are viral sequence variations in saliva and serum. Some authors have suggested that in some patients, serum and saliva paired specimens show different HCV genotypes (15). However, in this paper distribution of HCV genotypes in saliva specimens was not performed, because commercially available assays have not been manufactured for saliva samples analysis. HCVRNA has been detected in salivary glands (16) and it has been recently proved that HCV replicates in the epithelial cells from oral mucosa of anti-HCV-positive patients (17). The fact that HCV-RNA could persist in the saliva in spite of antiviral therapy provides a new evidence for the presence of compartmentalized extrahepatic replication. The genomic heterogeneity of HCV has major implications including viral persistence, pathogenesis of extra-hepatic involvement and response to antiviral therapy (18). Almost $50 \%$ of patients with chronic HCV infection have salivary gland lesions (lymphocytic capillaritis sometimes associated with lymphocytic sialoadenitis); a relationship between HCV-related sialadenitis and the persistence of HCV in saliva has not been demonstrated.

It has been proved that early changes in the HCV-RNA level may identify patient's chance of a sustained virologic response to CT (19). None of the three primary responders showing HCV clearance pattern 2a (delayed HCV serum clearance) became a sustained responder. However, four of the five patients with HCV clearance pattern 2 had either relapsed or previously not responded to IFN therapy, and it has been suggested that only $14 \%$ of the IFN non-responders become sustained responders with CT (20). The precise relationship of a clearance pattern of HCV in saliva and serum in response to appropriate antiviral therapy remains unclear. However, the present data would suggest that HCV may persist in the mouth despite clearance from blood.

In conclusion, HCV-RNA may need to be monitored in sites other than blood to confirm complete clearance of virus. However, this is a study of a relatively small number of patients, and a much more expanded work is required to confirm the present observations. HCV quantification and determination of viral sequence variations in paired serum and saliva samples could become useful in the selection of patients who could respond to treatment and establish response criteria to therapy.

\section{References}

1. Fried MW, Shindo M, Fong TL, Fox PC, Hoofnagle JH, Bisceglie M. Absence of hepatitis C viral RNA from saliva and semen of patients with chronic hepatitis C. Gastroenterology 1992; 102: 1306-8.

2. Takamatsu K, Koyanagi Y, Okita K, Yamamoto N. Hepatitis C virus in saliva. Lancet 1990; 336: 1515.

3. Hermida M, Ferreiro MC, Barral S, Laredo R, Castro A, Diz Dios P. Detection of HCV RNA in saliva of patients with hepatitis C virus infection by using highly sensitive test. J Virol Methods 2002; 101: 29-35.

4. Mastromatteo AM, Rapaccini GL, Pompili M, et al. Hepatitis C virus infection: other biological fluids than blood may be responsible for intrafamiliar spread. Hepatogastroenterology 2001; 48: 193-6.

5. EASL. International Consensus Conference on Hepatitis C. Consensus statement. J Hepatol 1999; 30: 956-61.

6. Roy KM, Bagg J, Bird GLA, et al. Serological and salivary markers compared with biochemical markers for monitoring interferon treatment for hepatitis C virus infection. J Med Virol 1995; 47: 429-34.

7. Poynard T, McHutchison J, Goodman Z, Ling MH, Albrecht J. Is an 'à la carte' combination interferon alfa-2b plus ribavirin regimen possible for the first line treatment in patients with chronic hepatitis C? Hepatology 2000; 31: 211-8.

8. Enríquez J, Gallego A, Torras X, et al. Retreatment for 24 vs. 48 weeks with interferon alfa-2b plus ribavirin of chronic hepatitis C patients who relapsed or did not respond to interferon alone. J Viral Hepat 2000; 7: 403-8.

9. Navazesh M, Christensen CM. A comparison of whole mouth resting and stimulated salivary measurement procedures. J Dent Res 1982; 61: 1158-62.

10. Fabris P, Infantolino D, Biasin MR, et al. High prevalence of HCV-RNA in the saliva fraction of patients with chronic hepatitis C but no evidence of HCV transmission among sexual partners. Infection 1999; 27: 86-91.

11. Belec L, Legoff J, Si-Mohamed A, et al. Mucosal humoral immune response to hepatitis C virus E1/E2 surface glycoproteins and HCV shedding in saliva and cervicovaginal fluids from chronically infected patients. $J$ Hepatol 2004; 38: 833-42.

12. Roy KM, Bagg J, Follett EA, Brewer A, Lowe GD. Hepatitis C virus in saliva of haemophiliacs patients attending an oral surgery unit. Br J Oral Maxillofac Surg 1996; 34: 162-5.

13. Pawlotsky J, Bouvier-Alias M, Hezode C, Darthuy F, Remire J, Dhumeaux D. Standardization of hepatitis C virus RNA quantification. Hepatology 2000; 32: 654-9. 
14. Roy KM, Bagg J, McCarron B. The effect of saliva specimen collection, handling and storage protocols on hepatitis C virus (HCV) RNA detection by PCR. Oral Dis 1999; 5: 123-7.

15. Roy KM, Bagg J, McCarron B, Good T, Cameron S, Pithie A. Predominance of HCV type 2a in saliva from intravenous drug users. J Med Virol 1998; 54: 271-5.

16. Arrieta JJ, Rodríguez-Iñigo E, Ortiz-Movilla N, et al. In situ detection of hepatitis C virus RNA in salivary glands. Am J Pathol 2001; 158: 259-64.

17. Carrozzo M, Quadri R, Latorre $\mathrm{P}$, et al. Molecular evidence that the hepatitis $\mathrm{C}$ virus replicates in the oral mucosa. J Hepatol 2002; 37: 364-9.

18. Gretch DR, Polyak SJ. (eds) The quasispecies nature of hepatitis $C$ virus: research methods and biological implications. In: Groupe Francais d'Etudes Molèculaires des Hépatites (GEMHEP). Hepatitis C virus: genetic heterogeneity and viral load. Paris, France: John Libbey Eurotext, 1997; 57-69.

19. Zeuzem S, Lee J, Franke A, et al. Quantification of the initial decline of the serum hepatitis C virus RNA and response to interferon alfa. Hepatology 1998; 27: 1149-56.

20. Di Bisceglie AM, Thompson J, Smith-Wilkaitis N, Brunt EM, Bacon BR. Combination of interferon and ribavirin in chronic hepatitis C: re-treatment of nonresponders to interferon. Hepatology 2001; 33: 704-7. 